\title{
2016 Usefulness of C-reactive protein in detecting higher risk patterns of myocardial infarction defined by contrast enhanced magnetic resonance imaging
}

\author{
Thomas N Martin*, Naveed Sattar, Bjoern Groenning, Galen Wagner, \\ Robin Weir, Simon Martin, Nirosha Gunitillake, Andrew Flapan and \\ Henry Dargie
}

Address: Glasgow University, Glasgow, UK

* Corresponding author

from I th Annual SCMR Scientific Sessions

Los Angeles, CA, USA. I-3 February 2008

Published: 22 October 2008

Journal of Cardiovascular Magnetic Resonance 2008, I0(Suppl I):A285 doi:10.I I86/I532-429X-I0-SI-A285

This abstract is available from: http://jcmr-online.com/content/I0/SI/A285

(c) 2008 Martin et al; licensee BioMed Central Ltd.

\section{Introduction}

A systemic inflammatory response has been reporeted in Acute Myocardial Infarction (AMI) with C-reactive protein (CRP) the most widely studied marker. Contrast enhanced Magnetic Resonance Imaging (ceMRI) allows accurate quantification of AMI size by delayed hyperenhancement (DE). CeMR also provides qualitative information on AMI characteristics, known to influence prognosis, such as microvascular obstruction (MVO).

\section{Purpose}

To investigate the relationship of inflammation to AMI characteristics.

\section{Methods}

80 consecutive patients with first AMI underwent ceMRI at a mean (SD) of 64 (23) hours from chest pain on a Siemens Sonata $1.5 \mathrm{~T}$ system using a phased array chest coil during breath-holds. Left ventricular ejection fraction (LVEF) was evaluated using a steady state free precession sequence. CeMRI was performed > 15 minutes after peripheral injection of $0.2 \mathrm{mmol} / \mathrm{kg}$ gadolinium-DTPA using a segmented gradient-echo inversion-recovery sequence for DE and MVO. Scans were assessed by 2 experienced observers and DE was planimetered manually. 23 patients were excluded based on ceMRI findings: DE in > 1 coronary territory $(\mathrm{n}=11)$; subendocardial sparing pat- tern of DE $(n=3)$; and absent DE $(n=9) .57$ patients (43 male) of mean (SD) age 59.8 (12.5) years were included in final analysis. CRP concentration was measured at time of ceMRI using an ultrasensitive double antibody sandwich ELISA.

\section{Results}

Patients with CRP > $10 \mathrm{mg} / \mathrm{dl}(75 \%)$ had a larger mean infarct size (40.1 vs. $14.2 \mathrm{~g}, \mathrm{p}=0.001$ ) and increased proportion of MVO (46.5 vs $14.3 \%, \mathrm{p}=0.03$ ). The difference in LVEF approached significance (54.4 vs. $60.5 \%$, $\mathrm{p}=$ $0.07)$. CRP correlates with DE $(\mathrm{r}=0.57, \mathrm{p}<0.001)$ and $\operatorname{LVEF}(\mathrm{r}=-0.28,<0.001)$. The area under the curve $(95 \%$ confidence interval) by ROC analysis was 0.8 (0.73-0.87) for presence of DE and 0.75 (0.62-0.87) for MVO. Using a cut off CRP value $>26 \mathrm{mg} / \mathrm{dl}$ the sensitivity for presence of MVO is 0.83 and specificity 0.63 , likelihood ratio 2.2 .

\section{Conclusion}

CRP measured within first few days of AMI has a moderate correlation with infarct size and LVEF by ceMRI. Moreover, a patient admitted with AMI with a CRP value of $>26$ $\mathrm{mg} / \mathrm{dl}$ is more than twice as likely to have microvascular obstruction. 